\title{
Q-switching of an all-fiber ring laser based on in-fiber acousto- optic bandpass modulator
}

\author{
G. Ramírez-Meléndez ${ }^{1}$, M. Bello-Jiménez ${ }^{1 *}$, O. Pottiez ${ }^{2}$, L. Escalante-Zarate ${ }^{2}$, R. López-Estopier ${ }^{1,3}$, B. Ibarra- \\ Escamilla $^{4}$, M. Durán-Sánchez ${ }^{3,4}$, E. A. Kuzin ${ }^{4}$ and M. V. Andrés ${ }^{5}$ \\ ${ }^{1}$ Instituto de Investigación en Comunicación Óptica (IICO), Universidad Autónoma de San Luis Potosí, Av. \\ Karakorum No. 1470 Lomas $4^{a}$ Secc., 78210 San Luis Potosí, México \\ Tel: $+52-444-825-0183$, Fax:+52-444-825-0198 \\ gustavo_r_melendez@hotmail.com,m.bello@cactus.iico.uaslp.mx,relopezes@conacyt.mx \\ ${ }^{2}$ Centro de Investigaciones en Óptica (CIO), Loma del Bosque No. 115, Col. Lomas del Campestre, León, \\ Guanajuato 37150, México \\ Tel: +52-477-441-42-00, Fax: +52-477-441-42-09 \\ pottiez@cio.mx,itrio@cio.mx \\ ${ }^{3}$ Consejo Nacional de Ciencia y Tecnología (CONACYT), Av. Insurgentes Sur No. 1582, Col. Crédito \\ Constructor, Del. Benito Juárez, México, D.F. 039040, México \\ Tel: $+52-55-5332-7708$ \\ ${ }^{4}$ Instituto Nacional de Astrofísica, Óptica y Electrónica (INAOE), Luis Enrique Erro No 1, Departamento de \\ Óptica, 72000 Puebla, México \\ Tel: +52-222-266-31-00, Fax: 247-25-80 \\ baldemar@inaoep.mx,manueld@inaoep.mx,ekuz@inaoep.mx \\ ${ }^{5}$ Universidad de Valencia, Departamento de Física Aplicada y Electromagnetismo, ICMUV, c/Dr. Moliner \\ 50, Burjassot, 46100 Valencia, Spain \\ Tel: +34-96-354-3338, Fax: +34-96-354-3146 \\ miguel.andres@uv.es \\ *Corresponding author: m.bello@cactus.iico.uaslp.mx
}

\begin{abstract}
Active Q-switching of an all-fiber ring laser by utilizing a novel in-fiber acousto-optic tunable bandpass filter (AOTBF) is reported. Transmission characteristics of the AOTBF are controlled by amplitude modulation of the acoustic wave, it exhibits a 3-dB power insertion loss, $0.91 \mathrm{~nm}$ of optical bandwidth, and $28 \mathrm{~dB}$ of nonresonant light suppression. Cavity loss modulation is achieved by full acousto-optic mode recoupling cycle induced by traveling flexural acoustic waves. When the acoustical signal is switched-on, cavity losses are reduced, and then laser emission is generated. Additionally, by changing the acoustic wave frequency, a wide wavelength tuning range of $30.7 \mathrm{~nm}$ is achieved from 1542 to $1572.7 \mathrm{~nm}$. Best Q-switched pulses were obtained at $1.1 \mathrm{kHz}$ repetition rate, with a pump power of $242 \mathrm{~mW}$, at the optical wavelength of $1569.4 \mathrm{~nm}$. A maximum pulse energy of $8.3 \mu \mathrm{J}$ at an average output power of $9.3 \mathrm{~mW}$ was achieved, corresponding to optical pulses of $7.8 \mathrm{~W}$ peak power and $1 \mu \mathrm{s}$ temporal width.
\end{abstract}

\section{Introduction}

Q-switched fiber lasers capable of producing short and high intense optical pulses are very attractive optical sources with important applications in many research fields. These include, for example, materials processing, medical engineering, remote sensing, optical communication and defense, among others [1-5]. The mechanism of short pulse emission is based on modulation of the Q-factor in the cavity [6, 7], which can be realized either passively or actively. In the case of passive Q-switching, cavity setups have a simple structure, and the repetition rate is only varied with the pump power of the gain medium [8,9]. In contrast, active Qswitching is accurately controlled by an electrical signal, which is used to trigger the modulator, allowing for more control over the cavity losses and the output pulse characteristics. Therefore, active Q-switching is the preferred technique in many different practical applications. 
One of the most common configurations of Q-switched fiber lasers relies on the use of fiber Bragg gratings (FBGs) as cavity mirrors. In this way, the tuning in wavelength of one of the FBGs is used to achieve active Q-switching. Examples of this technique can be found in refs. [10-14], where is reported the stretching of fiber Bragg gratings by magnetostrictive materials [10], by piezoelectric actuators [11, 12], or by the interaction of longitudinal acoustic waves with the FBG [13, 14]. Nevertheless, a common shortcoming is the restriction of wavelength emission, which is imposed by the Bragg wavelength of the fiber grating, and other shortcoming is the necessity of matching the wavelengths of the two FBG that define the Fabry-Perot cavity. These limitations have motivated the development of Q-switching devices that avoid the inclusion of fixed filtering elements in the laser cavity. In this respect, in-fiber acousto-optic (AO) devices can be designed to perform a bandpass operation with a widely tunable spectral response [15-19]. In a previous work, we reported the operation of an efficient all-fiber acousto-optic tunable bandpass filter (AOTBF) that combines the dynamic properties of flexural acoustic waves with a fiber core-mode blocker (CMB) [20]. With the objective of expanding the application of the AOTBF to perform active Q-switching, the acoustic wave is modulated by a rectangular pulse of variable repetition frequency and duty cycle. This modulation produce on-off periods of the acoustic wave being imposed to the fiber, which results in a modulation of the cavity losses and the generation of Q-switched optical pulses.

In this paper we demonstrate active Q-switching of an all-fiber ring laser by utilizing an efficient in-fiber AOTBF. As opposed to the work reported by Kang et al., where a wavelength tunable fiber ring laser incorporating an all-fiber acousto-optic tunable filter and a CMB is reported [21], here a distinguishable feature of the proposed AOTBF is the inclusion of a CMB composed of a tiny section of coreless optical fiber. The merits of this AOTBF are reliability, superior rejection efficiency and simplicity in fabrication. Additionally, we also report the potential of the AOTBF to be utilized as a Q-switched element in a fiber laser. Compared to other all-fiber approaches, this active Q-switching technique presents three main advantages: (i) high modulation depth $(28 \mathrm{~dB})$, which is more than $10-\mathrm{dB}$ in depth that conventional all-fiber AO attenuators [22-24], (ii) intrinsic spectral filtering (linewidth $\sim 1 \mathrm{~nm}$ ), this prevents lasing at unwanted wavelengths and can be tuned in a broad wavelength range $(30 \mathrm{~nm})$, and (iii) being an all-fiber device, it can handle high power. We should mention that some characteristics of the present device, as insertion losses and the time response, could be improved further, as we will discuss in the following sections.

\section{The acousto-optic bandpass filter}

A schematic of the acousto-optic tunable bandpass filter is depicted in Fig.1. It consists of a core-mode blocker composed of a tiny section of coreless optical fiber (Thorlabs FG125LA) spliced between two sections of standard single-mode fibers (SMF-28) with length $L_{1}$ and $L_{2}$, respectively. The length $d$ of the CMB is calculated for the light leaving section $L_{I}$ to be expanded up to the outer surface at the front face of the following single-mode fiber. Assuming an effective index of 1.446 for the $L P_{01}$ mode and a refractive index of 1.444 for the coreless fiber, the distance $d$ is calculated to be $1.185 \mathrm{~mm}$. With this configuration, the fundamental mode is strongly attenuated in a broad band that extends from 1500 to $1600 \mathrm{~nm}$ with a maximum attenuation of $-28 \mathrm{~dB}$ at $1565 \mathrm{~nm}$. The AOTBF is completed with the radio frequency (RF) source, a piezoelectric disk (PD), and an aluminum horn. The horn is attached to the piezoelectric disk, and it focuses the vibrations into the optical fiber through its tip, which is glued to an uncoated optical fiber to prevent the attenuation of the acoustic wave. In order to avoid acoustic reflections, the acoustic wave is damped at both ends of the filter structure.

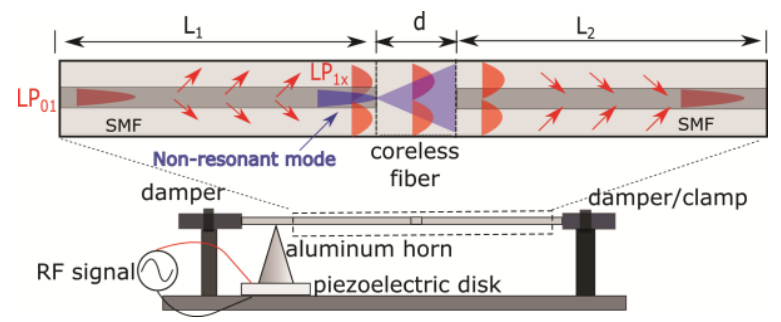


Fig.1. Experimental setup of the AOTBF based in a coreless optical fiber CMB.

When a flexural acoustic wave propagates along the uncoated optical fiber, the acoustic field produces a periodical perturbation of the fiber, which leads to an intermodal resonant coupling between the fundamental core mode and some specific cladding modes of the SMF [25, 26]. This AO interaction can be seen as the dynamic counterpart of a conventional long-period grating (LPG), whose transmission properties can be controlled dynamically by the amplitude and frequency of the acoustic wave. In our approach, the section of fiber $L_{1}$ was set to $24 \mathrm{~cm}$ in length, allowing the $L P_{01}$ mode to be coupled to a cladding mode prior the CMB, whereas section $L_{2}$ is used to couple the light back to the core mode. This reinsertion of energy results in the appearance of a transmission peak in the spectrum, with an amplitude that remains fixed whenever the traveling acoustic wave is excited. Under this scheme the device operates as a bandpass filter, and wavelengths that do not fulfill the phase-matching condition are strongly attenuated. By following a similar process to that reported in reference [20], the response of the AOTBF is optimized with an asymmetric configuration that takes into account the attenuation of the acoustic wave. For the set of experiments reported here, the length of section $L_{2}$ was adjusted to find the optimal interaction length for a maximum reinsertion of energy in the $1550 \mathrm{~nm}$ region. Section $L_{2}$ was selected to be $48.5 \mathrm{~cm}$ in length, giving a total length of $\sim 72.5$ $\mathrm{cm}$ for the AO device. The transmission characteristics of the AOTBF were investigated by illuminating the device with a superluminescent diode light source (SLD), that provides an optical spectrum from 1500 to $1600 \mathrm{~nm}$, and detecting the transmitted light with an optical spectrum analyzer. The strongest mode-coupling was found at the acoustic frequency $\left(f_{a}\right)$ of $2.430 \mathrm{MHz}$ for a voltage applied to the piezoelectric disk $\left(V_{P D}\right)$ of $24 \mathrm{~V}$ (whenever we refer to voltages, it is a peak-to-peak measurement). Fig. 2(a) shows the spectral response, in which $L P_{01}$ to $L P_{11}$ and $L P_{01}$ to $L P_{12}$ intermodal couplings were observed with a wavelength separation of $\sim 50 \mathrm{~nm}$. The maximum transfer of energy corresponds to the $L P_{01}$ to $L P_{12}$ coupling located at the resonant optical wavelength $\left(\lambda_{R}\right)$ of $1568.8 \mathrm{~nm}$, with a measured 3-dB optical bandwidth of $0.91 \mathrm{~nm}$ and a minimum insertion loss of $-3 \mathrm{~dB}$. The period of the acoustic LPG is estimated to be $682 \mu \mathrm{m}$, obtained from the dispersion relation for a flexural acoustic wave on a cylindrical $\operatorname{rod} \Lambda=\sqrt{ }\left(\pi R C_{\text {ext }} / f_{a}\right)$, where $\Lambda$ is the acoustic wavelength, $R$ is the fiber radius and $C_{e x t}$ is the speed of the extensional wave $\left(5760 \mathrm{~ms}^{-1}\right.$ for fused silica). The experimental characterization of the AO resonances is shown in Fig. 2(b) for a range of resonant optical wavelengths between 1520 and $1600 \mathrm{~nm}$. Working in the frequency range from 2 to $2.8 \mathrm{MHz}, L P_{01}$ to $L P_{11}$, $L P_{01}$ to $L P_{12}$, and $L P_{01}$ to $L P_{13}$ intermodal couplings are observed. The dashed line indicates the strongest mode coupling at the acoustic frequency of $2.430 \mathrm{MHz}$. Fig. 2(c) shows the measured transmission spectrum corresponding to the $L P_{01}$ to $L P_{12}$ intermodal coupling. In order to provide a detailed description of the AO response, Fig. 2(d) illustrates the variations of the peak transmission for the $L P_{01}$ to $L P_{12}$ resonance as a function of the applied frequency and the resonant optical wavelength. For this measurement a tunable laser diode $(1520-1570 \mathrm{~nm})$ was used to select the resonant optical wavelength and the transmitted light was measured with a detector and a standard oscilloscope. As expected, different frequencies produce a different transmission response, however, no sharp lines of resonances were observed in the range from 2.690 to 2.425 $\mathrm{MHz}$, measured in steps as small as $5 \mathrm{kHz}$. The lack of flatness is a feature quite common in this kind of AO devices, and its origin is the nonflat frequency response of the piezoelectric disk. The best performance of the AOTBF was found between 1550 and $1570 \mathrm{~nm}$, corresponding to the frequency range from 2.525 to $2.425 \mathrm{MHz}$. 

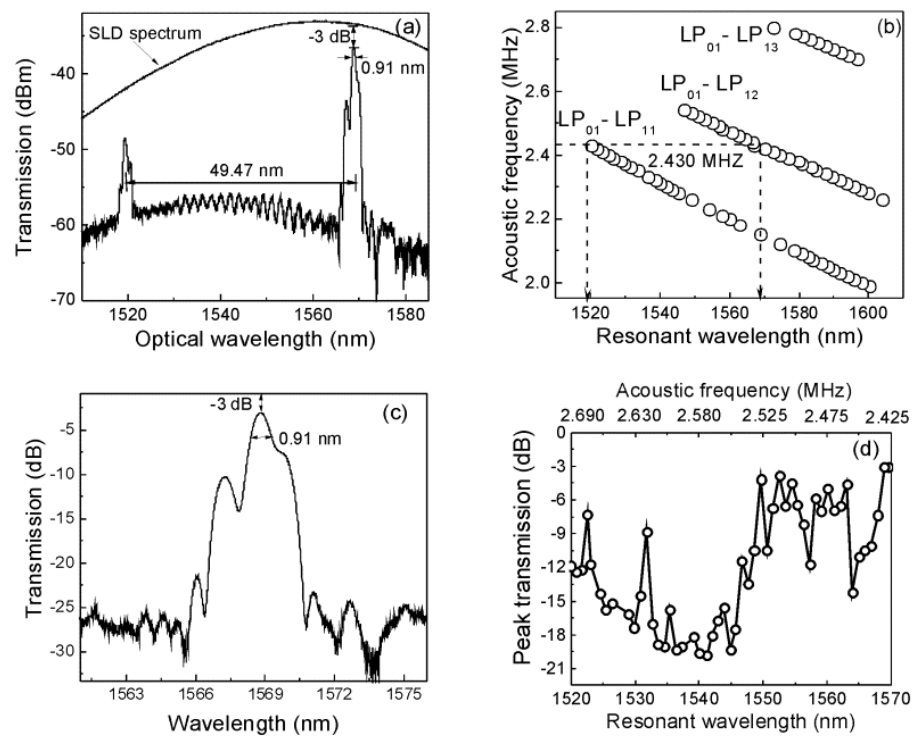

Fig. 2. (a) Spectral response of the AOTBF at the acoustic frequency of $2.430 \mathrm{MHz}$. (b) Tuning characteristics of the AOTBF: acoustic wave frequency vs resonant optical wavelengths. The dashed line indicates the strongest mode coupling around the $1550 \mathrm{~nm}$ region. (c) Transmission spectrum corresponding to the $L P_{0 I}$ to $L P_{12}$ intermodal coupling. (d) Variations of the peak transmission for the $L P_{01}$ to $L P_{12}$ resonance as a function of the applied frequency and the resonant optical wavelength.

The time response of the AOTBF is shown in Fig. 3. For this experiment, the AO device was illuminated with a tunable laser diode, whose emission wavelength was tuned to match the resonant optical wavelength, and a square voltage was applied to modulate the transmission. The frequency of the RF signal is $2.430 \mathrm{MHz}$, allowing resonant intermodal coupling at the optical wavelength of $1568.8 \mathrm{~nm}$. As illustrated in Fig. 3(a), the positive switching time, $\mathrm{i}$ e., the time that the signal takes to change from zero to a maximum steady state, is about $181 \mu \mathrm{s}$. This time corresponds to the time that the acoustic wave takes to travel along the section of fiber $L_{2}$, where the reinsertion of energy takes place. A delay of $110 \mu$ s between the electrical and the optical signals is also observed, it corresponds to the time that the acoustic wave takes to travel from the piezoelectric disk to the end of section $L_{l}$, where the CMB is located. Fig. 3(b) shows the behavior of the device when the modulated signal is switched off, the transmittance signal decays from the highest transmission to zero at the time of $96 \mu \mathrm{s}$. This decay time corresponds to the time that the acoustic wave takes to travel along section $L_{l}$ of the AOTBF, eliminating the coupling between the fundamental core mode and the cladding mode.
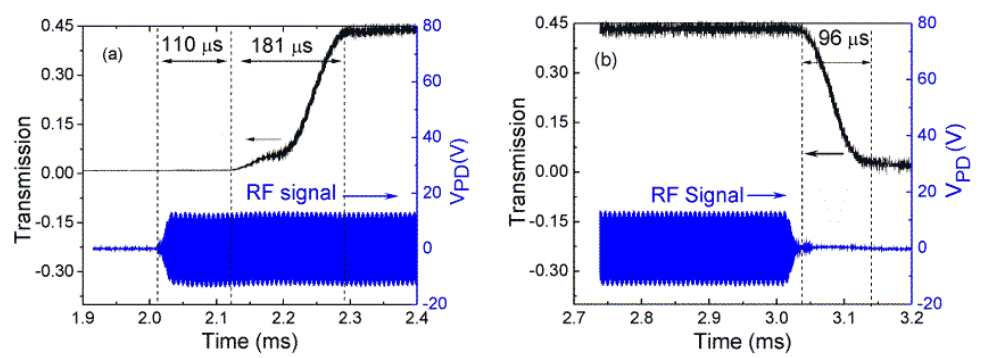

Fig. 3. Time response of the AOTBF. (a) Rise time of the optical signal compared with the RF signal. (b) Decay time of the optical signal when the RF signal is switched off.

\section{The Q-switched all fiber laser}


A schematic setup of the Q-switched all-fiber ring laser is illustrated in Fig. 4. The gain medium is provided by a 3-m long Er/Yb co-doped double-cladding fiber (EYDCF, CorActive, DCF-EY-10/128), exhibiting a core absorption of $\sim 85 \mathrm{~dB} / \mathrm{m}$ in the $1535 \mathrm{~nm}$ region. The active fiber is pumped through a $(2+1) \times 1$ fiber combiner by a pigtailed multimode laser diode emitting at $975 \mathrm{~nm}$, providing a maximum pump power of 4 $\mathrm{W}$. The $\mathrm{Er} / \mathrm{Yb}$ fiber is connected to one input port of a symmetrical fiber coupler (50/50). One output port of the coupler is used to provide the output light pulses, and the remaining port is connected to a polarization controller (PC) to allow fine adjustment of polarization. The AOTBF is connected after the PC, and is followed by a fiber isolator to force unidirectional operation within the laser cavity. Finally, the ring cavity is closed by connecting the isolator to the signal port of the combiner.

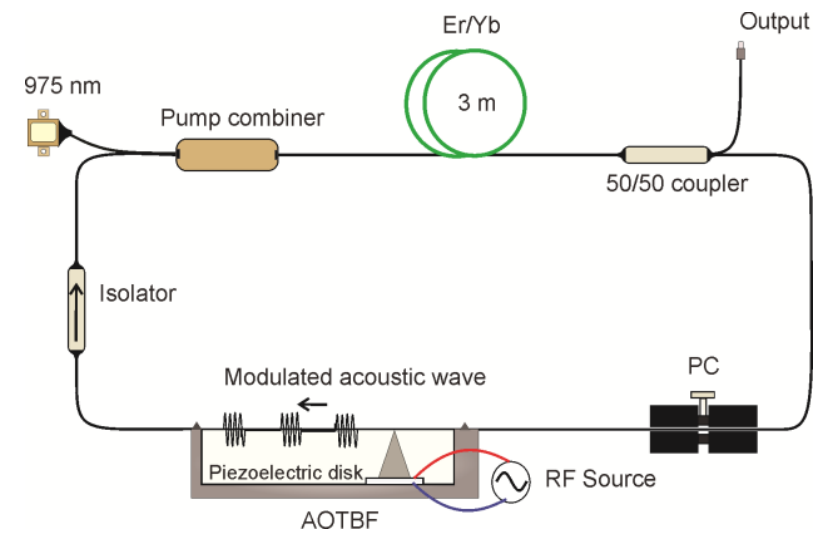

Fig. 4. Schematic setup of the Q-switched all-fiber ring laser.

Initial measurements of laser performance were realized in the continuous-wave regime. The acoustic parameters, which are controlled by the RF frequency and voltage applied to the piezoelectric disk (PD), were selected to be the same as in Fig. 2, i e., $2.430 \mathrm{MHz}$ and $24 \mathrm{~V}$. Thus, the AOTBF is operated within the conditions of strongest mode coupling. Fig. 5(a) shows the CW laser emission spectra (red line). The laser line was narrower than $30 \mathrm{pm}$, which is the resolution limit of our measurement system. A signal-to-noise ratio as high as $63 \mathrm{~dB}$ was obtained when pump power reaches the value of $160 \mathrm{~mW}$. No laser emission was observed when the RF signal generator was off even when the pump power reached its maximum value of 4 W. Tunability of the laser emission was demonstrated by measuring the wavelength shift as a function of the acoustic frequency, as shown in Fig. 5(a). For this measurement, the pump power was maintained fixed at 160 $\mathrm{mW}$ and the acoustic frequency was varied to obtain laser emission in the $1550 \mathrm{~nm}$ region. The first laser emission appears at the acoustic frequency of $2.3997 \mathrm{MHz}$, corresponding to an optical wavelength of 1572.7 $\mathrm{nm}$. Subsequently, a fine tuning from 1572.7 to $1542 \mathrm{~nm}$ was observed as frequency was increased. The rate of change was measured to be $-0.18 \mathrm{~nm} / \mathrm{kHz}$, and the shortest wavelength emission corresponds to the acoustic frequency of $2.570 \mathrm{MHz}$. A tuning range over $30.7 \mathrm{~nm}$ is achieved in our setup. The power difference between maximal and minimal lasing lines was measured as $20 \mathrm{~dB}$. This nonflat response is essentially the same to that reported in Fig. 2(d), and its origin is the nonflat frequency response of the PD. Laser emission below $1542 \mathrm{~nm}$ was not possible to observe since the low transmission response of the AOTBF and the strong core absorption of $85 \mathrm{~dB} / \mathrm{m}$ at $1535 \mathrm{~nm}$ of the Er/Yb fiber. Fig. 5(b) plots the output power as a function of applied pump power, under the condition of maximum transmission of the AOTBF $(2.430 \mathrm{MHz}$ and $24 \mathrm{~V})$. The efficiency and the threshold were $15 \%$ and $120 \mathrm{~mW}$, respectively.
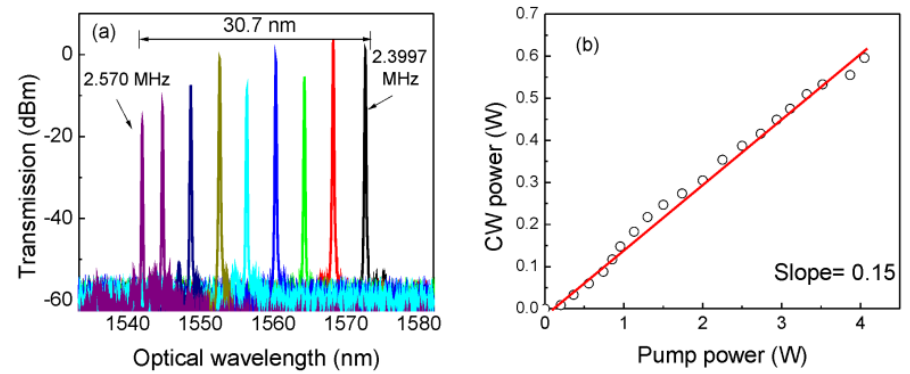
Fig. 5. (a) Tunable operation of the fiber laser at constant pump power $(160 \mathrm{~mW})$ and different RF frequency (see the label for the traces). (b) Output power as a function of applied pump power at constant RF signal $(2.430 \mathrm{MHz})$. In both cases the voltage applied to the piezoelectric disk was $24 \mathrm{~V}$.

Q-switching was performed by modulation of the RF signal with a rectangular wave of variable frequency and duty cycle. This modulation produced on-off periods of the acoustic wave being imposed to the fiber, which results in a modulation of the cavity losses at the laser emission wavelengths. Fig. 6(a) shows the pulse train emitted by the laser at a repetition rate of $1.1 \mathrm{kHz}$ at the optical wavelength of $1569.4 \mathrm{~nm}$. The frequency of the RF signal was $2.426 \mathrm{MHz}$, and its amplitude is modulated by the rectangular pulse signal (shown in blue). When the acoustic signal is turned off, the cavity has high losses, so no lasing occurs and the energy provided by the pumping mechanism is stored; when the acoustic signal is turned on, the cavity losses at the selected wavelength are reduced and the laser pulse builds up. The duty cycle of the RF signal was adjusted to optimize the amplitude and stability of the pulses. Fig. 6(b) shows a close-up view of one Q-switched pulse, it exhibits a width of $1 \mu \mathrm{s}$ and a peak power of $7.8 \mathrm{~W}$. A detailed inspection of the Q-switched pulse reveals a splitting into smaller pulses, whose separation coincides with the frequency mode-spacing of the laser cavity. This apparent modelocking response has been previously reported in both, passively and actively Q-switched fiber lasers [27]. A time separation of $93 \mathrm{~ns}$ between the sub-pulses was measured, this time agrees with the round-trip time of the cavity $\sim 20 \mathrm{~m}$. The effect of high pump power is illustrated in Fig. 6(c), where singlepulse operation is limited by satellite pulses [28]. This condition restricts the Q-switched operation to a maximum pump power of $242 \mathrm{~mW}$.
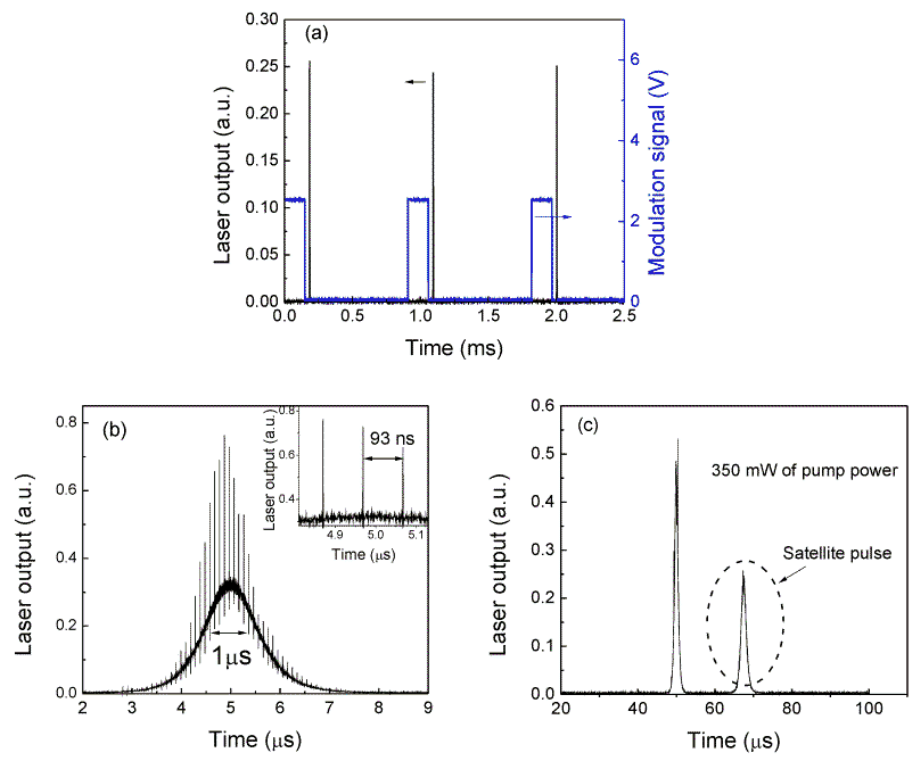

Fig. 6. (a) Train of Q-switched pulses generated with a pump power of $242 \mathrm{~mW}$ at a repetition rate of 1.1 $\mathrm{kHz}$. The blue line corresponds to the modulation signal. (b) Optical pulse shape. The inset shows a zoom-in view of generated sub-pulses. (c) Appearance of satellite pulses at pump powers above $242 \mathrm{~mW}$.

Fig. 7 illustrates the behavior of the Q-switched laser as a function of the pump power and repetition rate. By maintaining fixed the repetition rate at $1.1 \mathrm{kHz}$, the peak power of the pulses increases with pump power, and there is a corresponding reduction of pulse width, see Fig. 7(a). The pulse width decreases from $3.03 \mu$ s at the lowest pump power, down to $1 \mu \mathrm{s}$ at the maximum pump power available. Pulses of $7.8 \mathrm{~W}$ of peak power were generated by pumping with $242 \mathrm{~mW}$. Fig. 7(b) shows the effect of increasing the repetition rate on the Q-switched pulses. The peak power starts to fall off and the pulse width increases for frequencies above 1.1 $\mathrm{kHz}$, due to the recovery time of the Er/Yb system. Pumping with $242 \mathrm{~mW}$, the pulse width increases from 1 $\mu$ s for the strongest peaks generated at low frequency, up to $4.4 \mu$ s at the highest repetition rate achieved, 4.5 $\mathrm{kHz}$. 

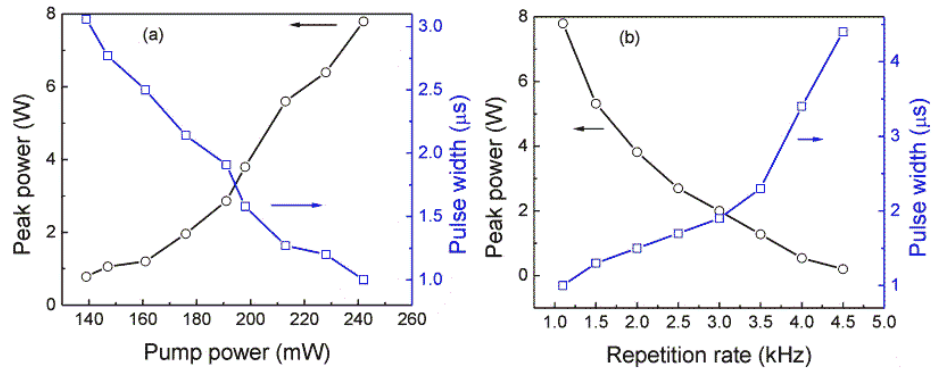

Fig. 7. (a) Peak power and pulse width as a function of the pump power at a repetition rate of $1.1 \mathrm{kHz}$. (b) Peak power and pulse width as a function of the repetition rate for a pump power of $242 \mathrm{~mW}$.

From the point of view of implementation, our approach based on a tunable AO bandpass filter is well suited to modulate the Q-factor of the cavity and to generate Q-switching. The AOTBF can be dynamically controlled by the acoustic wave, providing a fine-tuning of laser emission in a range from 1542 to $1572.7 \mathrm{~nm}$. Additionally, with the use of this modulator, laser operation is simplified since no additional filtering devices are necessary due to the bandpass characteristics of the modulator. Further improvements in efficiency and interaction length could be possible by reducing the length of the fiber cavity and also by improving the time response of the $\mathrm{AO}$ bandpass filter.

\section{Conclusions}

We have reported an all-fiber acousto-optic tunable bandpass modulator suitable for Q-switching applications. The AO device is based on a CMB, composed of a tiny section of coreless optical fiber $(1.185 \mathrm{~mm})$ spliced between two sections of standard single-mode fibers. The bandpass characteristic of our device provides the advantage of avoiding the use of Bragg gratings, and provides a fine tuning of wavelength emission at a rate of $-0.18 \mathrm{~nm} / \mathrm{kHz}$. A wide tuning-range from 1542 to $1572.7 \mathrm{~nm}$ was obtained. Q-switching operation was performed by controlling the transmission characteristics of the AOTBF. Active Q-switching can be operated from $1 \mathrm{kHz}$ to $4.5 \mathrm{kHz}$ and the pulse width changes from $1 \mu$ s to $4.4 \mu \mathrm{s}$, respectively. Using the maximum pump power of $242 \mathrm{~mW}$, optical pulses with $7.8 \mathrm{~W}$ of peak power were obtained at $1.1 \mathrm{kHz}$ of repetition rate.

\section{Acknowledgements}

This work has been financially supported by CONACyT "Fronteras de la ciencia" grant 2016-01-2438 and CONACyT Grant 222476.

\section{References}

1. S.Gräf, G. Staupendhal, A. Krämer, F. A. Müller: Opt. Laser Eng. 66, 152(2015)

2. I. Rhode, J. M. Mash, D. Theisen-Kunde, M. Marczynski-Bühlow, R. Bomben Quaden, G. Lutter, R. Brinkmann: J. Card. Surg. 30, 157(2015)

3. M.C. Lee, Ch. S. Chang, Y. L. Huang, Sh. L. Chang, Ch. H. Chang, Y. F. Lin, S. Hu: Lasers Med. Sci. 30, 159(2015)

4. A.V. Fedorov, A. A. Fomichev, M. E. Doroshenko: CLEO OSA paper STh4J.5 (2016)

5. F. Ashraf, M. Talaat: Opt. Laser Technol. 64, 124(2014)

6. E. M. Sholokhov, A.V. Marakulin, A. S. Kurkov, V.B. Tsetkov, Laser Phys. Lett. 8, 382 (2011)

7. M. Delgado Pinar, A. Diez, J. L. Cruz, M. V. Andrés: Laser Phys. Lett. 6, 139(2009)

8. S. M. Kobstev, S. V. Kukarin, Y. S. Fedotov: Laser Phys. 18, 1230(2008)

9. A. S. Kurkov, E. M. Sholokhov, O. I. Medvedkov: Laser Phys. Lett. 6, 135(2009)

10. P. Pérez-Millán, A. Díez, M. V. Andrés: Opt. Express 13, 5046(2005)

11. N. A. Russo, R. Duchowicz, J. Mora, J. L. Cruz, M. V. Andrés: Opt. Commun. 210, 361(2002)

12. T. Imai, T. Komukai, T. Yamamoto, M. Nakawaza: Electron. Commun. Jpn. Part 2(80), 12(1997) 
13. C. Cuadrado-Laborde, M. Delgado-Pinar, S. Torres-Peiró, A. Díez, M. V. Andrés: Opt. Commun. 274, 407(2007)

14. M. Delgado-Pinar, D. Zalvidea, A. Díez, P. Pérez-Millán, M. V. Andrés: Opt. Express 14, 1106(2006)

15. C. Cuadrado-Laborde, M. Bello-Jiménez, A. Díez, J. L. Cruz, M. V. Andrés: Opt. Lett 39, 68(2014)

16. T. Dimmick, D. A. Satorius, G. L. Burdg: in Optical Fiber Communication Conference and International Conference on Quantum Information (OSA) paper WJ3 (2001)

17. D. A. Satorius, T. E. Dimmick, G. L. Burdge: IEEE Photon. Technol. Lett. 14, 1324(2002)

18. Y. Jung, S. Choi, Y. S. Jeong, S. B. Lee, J. W. Yu, K. Oh: CLEO OSA paper CThMM6(2004)

19. K. J. Lee, D. II Yeom, B. Y. Kim: Opt. Express 15, 2987(2007)

20. G. Ramírez-Meléndez, M. Bello-Jiménez, O. Pottiez, M. V. Andrés: IEEE Photon. Technol. Lett. 29, 1015(2017)

21. M. S. Kang, M. S. Lee, J. Ch. Yong, B. Y. Kim: J. Ligthwave Technol. 24, 1812(2006)

22. D. II Yeom, H.S. Kim, M. S. Kang, H. S. Park, B. Y. Kim: IEEE Photon. Technol. Lett. 17, 2646(2005)

23. F. Abrishamian, S. Nagai, S. Sato, M. Imai: Opt. Quant. Electron. 40, 665(2008)

24. D. Zalvidea, N. A. Russo, R. Duchowicz, M. Delgado-Pinar, A. Díez, J. L. Cruz, M. V. Andrés: Opt. Commun. 244, 315(2005)

25. S. D. Lim, J. G. Kim, K. Lee, S. B. Lee, B. Y. Kim: Opt. Lett. 11, 389(1986)

26. T. A. Birks, P. St. Russel, D. O. Culverhouse: J. Lightwave Technol. 14, 2519(1996)

27. T. Dimmick, D. A. Satorius, G. L. Gurdge: IEEE Photon. Technol. Lett. 14, 1324(2002)

28. S. Zhang, F. Lu, J. Wang: Microw. Opt. Technol. Lett. 49, 2183(2007) 